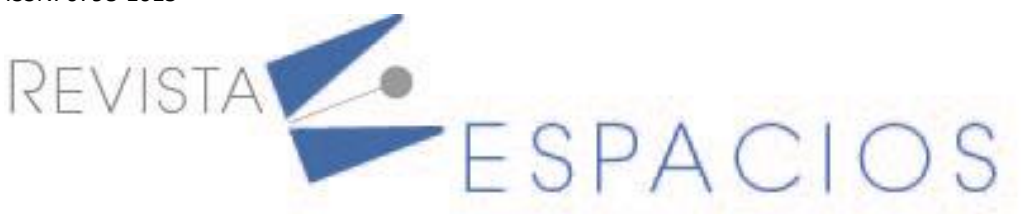

\title{
Diseño e Implementación de aplicación web para recolección y procesamiento de datos del Modelo TAG
}

\section{Design and Implementation of Web Application for Data Collection and Processing of the TAG Model}

\author{
AGUAS NÚÑEZ, Roberto L. ${ }^{1}$ \\ BUELVAS FERREIRA, Karen $\mathrm{G}^{2}$ \\ PÉREZ CAMPO, Andrés F. ${ }^{3}$
}

\begin{abstract}
Resumen
Este trabajo presenta el proceso de diseño e implementación de una aplicación web para valorar el nivel de ubicuidad en las funciones misionales de una Institución de Educación Superior (IES) mediante el modelo TAG (Tecnología, Aprendizaje, Gestión) y los resultados de su puesta en funcionamiento en dos instituciones de diferentes niveles de complejidad.

Palabras clave: tecnología, aprendizaje, gestión, valoración, modelo, educación superior, aplicación web.

Abstract

This paper presents the process of designing and implementing a web application to assess the level of ubiquity in the mission functions of a Higher Education Institution through the Technology, Learning and Management model (TAG in its Spanish and the results of its implementation in two institutions of different levels of complexity.

Keywords: technology, learning, management, assessment, model, higher education, web application.
\end{abstract}

\section{Introducción}

Con la evolución de la tecnología comienzan a surgir nuevas formas de comunicarse y de crear, acceder y difundir el conocimiento, lo que facilita su proceso de democratización sin importar el momento o el lugar en el que se encuentren las personas. Este nuevo escenario no sólo implica una transformación en las formas de enseñar y aprender, sino también en la manera como las Instituciones de Educación Superior deben gestionar sus funciones para responder a los constantes retos del entorno y, en general, a todas las demandas y desafíos que impone la Sociedad del Conocimiento.

En esa dirección, es necesario que las organizaciones educativas y en particular las Instituciones de Educación Superior (IES) se preparen para emprender este paradigma educativo y dispongan sus capacidades técnicas, de formación y organizacionales con miras a los nuevos desafíos y a la rápida evolución e irrupción de la tecnología

\footnotetext{
${ }^{1}$ Profesor. Facultad de Ingeniería. Universidad del Magdalena. raguas@unimagdalena.edu.co

${ }^{2}$ Ingeniero de Sistemas. Centro de Tecnologías Educativas y Pedagógicas. Universidad del Magdalena. kbuelvas@unimagdalena.edu.co

${ }^{3}$ Ingeniero de Sistemas. Centro de Tecnologías Educativas y Pedagógicas. Universidad del Magdalena. andresperezfc@unimagdalena.edu.co
} 
en todos los ámbitos de la humanidad. Es en este contexto en el que toma relevancia una tendencia pedagógica inspirada en los fundamentos de la computación ubicua enunciados por Weiser (1993a) (1993b) (1994): El aprendizaje ubicuo (U-Learning).

Consecuentemente, surge una definición de aprendizaje ubicuo entendido como aquel que puede llevarse a cabo en cualquier momento y lugar y, además, que puede ser realizado por cualquier persona indistintamente de su ocupación, formación, nivel de conocimiento, etc. (Cope \& Kalantzis, 2009). Adicionalmente, en este nuevo paradigma de educación se resalta la posibilidad de que las personas no solo consuman los conocimientos sino que aporten a su construcción con base en las sus propias experiencias.

Luego de una revisión bibliográfica, se identificaron factores relevantes para que los Centros educativos adopten metodologías para el aprendizaje involucrando la tecnología y esto, a su vez, permitió determinar que la implementación debe abordarse desde diferentes enfoques y actuando en distintos aspectos que permitan la cobertura de todos los procesos dentro de una IES. Considerando esto, diversos autores tales como: Zea, Trujillo, Atuesta y Foronda (2005), Salinas (2004), Duart y Lupiañez (2004) y Williams (2003) han realizado aportes significativos en la identificación de dimensiones que permiten conceptualizar, diseñar y, en general, construir entornos educativos con base tecnológica.

En el caso de Zea, Trujillo, Atuesta y Foronda (2005), se establecen diferencias "entre los aspectos técnicos, institucionales y pedagógicos" con el propósito de disminuir la posibilidad de que la construcción del conocimiento sea mermada con la aplicación de las metodologías de e-learning. Además, en Zea, Atuesta y González (2000) se señalan como áreas de mayor relevancia en lo que concierne a la formación profesoral para entornos virtuales las siguientes: pedagógica, didáctica, tecnológica y de gestión. Finalmente, en Zea y Atuesta (2007) se determinan que los aspectos sobre los cuales se fundamenta la construcción de una comunidad educativa interativa son la tecnología, la pedagogía y el sentido de comunidad.

Simultáneamente Salinas (2004), puntualiza tres elementos necesarios al momento de diseñar ambientes virtuales de aprendizaje empleando las perspectivas: pedagógica, organizacional y tecnológica; Duart y Lupiañez (2004) afirman que el e-learning surge de la interacción entre modelos educativos, modelos tecnológicos y modelos organizativos. Por último, Williams (2003) diserta sobre la educación superior a distancia estableciendo cuatro grupos de competencias necesarias para el aprendizaje bajo este concepto: comunicación e interacción, gestión, tecnología, y aprendizaje e instrucción, en total identifica 30 compentencias asociadas al aprendizaje a distancia que se distribuyen en los grupos ya enunciados.

Evidentemente, con base en estos análisis es posible afirmar que la implemtación de comunidades y entornos de aprendizaje basados en tecnología requiere ser abordada desde diferentes dimensiones de la organización haciendo especial énfasis en la tecnología, el escenario pedagógico (enseñanza y aprendizaje) y la gestión organizacional. Sin embargo, los autores antes revisados no proponen formas que permitan evaluar el cumplimiento de dichas dimensiones o perspectivas.

Es así, como surge el modelo teórico TAG (Tecnología, Aprendizaje y Gestión), el cual posibilita establecer el nivel de ubicuidad de sus procesos misionales de una IES bajo la perspectiva de los principios del Aprendizaje Ubicuo a partir de la valoración de las dimensiones de tecnología, aprendizaje y gestión (Aguas, 2018).

Bajo este marco conceptual el propósito de este trabajo es evidenciar el proceso de diseño e implementación de una aplicación web para la recolección y procesamiento de datos de las IES que sean objeto de aplicación del modelo TAG. 


\subsection{Caracterización del Modelo TAG}

A continuación, se presentan los aspectos generales del modelo TAG y se especifica su objetivo, ámbito de aplicación, usuarios y replicabilidad:

Cuadro 1

Descripción del Modelo TAG

\begin{tabular}{|c|c|}
\hline Aspecto & Descripción \\
\hline Objetivo del modelo & $\begin{array}{l}\text { Determinar el nivel de ubicuidad de los procesos misionales de una Institución de } \\
\text { Educación Superior (IES) con base en las dimensiones: Tecnología, Aprendizaje y } \\
\text { Gestión. }\end{array}$ \\
\hline Ámbito de aplicación & $\begin{array}{l}\text { Instituciones de Educación Superior de orden nacional o internacional } \\
\text { (considerando el contexto legal y normativo en cada caso), en especial si cuentan } \\
\text { con estructura organizacional definida y su funcionamiento obedece a un sistema } \\
\text { de procesos modelado e implementado. }\end{array}$ \\
\hline Objeto de estudio & $\begin{array}{l}\text { Verificar la aplicabilidad de los fundamentos del aprendizaje ubicuo (U-learning) } \\
\text { en los procesos misionales de las IES. }\end{array}$ \\
\hline Usuarios finales & $\begin{array}{l}\text { Personas con la potestad amplia y suficiente para decidir los destinos de las IES, } \\
\text { como también, investigadores y cualquier organización nacional o internacional } \\
\text { con interés en la aplicabilidad del U-Learning en las IES. }\end{array}$ \\
\hline Replicabilidad & $\begin{array}{l}\text { En todas las Instituciones de Educación Superior de orden nacional o } \\
\text { internacional (considerando el contexto legal y normativo en cada caso), en } \\
\text { especial si cuentan con estructura organizacional definida y su funcionamiento } \\
\text { obedece a un sistema de procesos modelado e implementado. }\end{array}$ \\
\hline
\end{tabular}

Fuente: elaboración propia a partir de (Aguas, 2018)

\section{Metodología: Diseño y desarrollo de la aplicación Web para el modelo TAG}

Para la construcción de la aplicación web se escoge la metodología de desarrollo de software denominada programación extrema (extreme programming - XP) por cuatro razones fundamentales: 1) está enfocada a la escritura y prueba de código, por lo que la creación de un producto o un prototipo se convierte en el principal objetivo en el desarrollo del proyecto; 2 ) los proyectos desarrollados con esta metodología están pensados para llevarse a cabo con un número reducido de personas en el equipo de trabajo; 3) con programación extrema se hace necesario la realización constante de pruebas que ayuden en la identificación de errores y la ejecución de una buena implementación de la aplicación; 4) al ser una metodología de desarrollo ágil se espera que el proceso de creación de la propuesta del proyecto y la implementación de este, estén siempre conectados y así cumplir con los objetivos deseados en la propuesta (Fojtik, 2011).

En esta dirección, se ejecutaron las cuatro actividades que propone XP, las cuales se detallan a continuación.

\subsection{Planificación}

En esta etapa resultó fundamental realizar un proceso de apropiación de los fundamentos y mecanismos de valoración de las tres dimensiones del modelo TAG desarrolladas por Aguas (2018).

En dicho modelo cada dimensión se define a partir de las categorías, propiedades, indicadores y aspectos a evaluar que la componen. Dado que TAG valora en general todos los aspectos de una IES es un modelo complejo que distribuye dentro de sus tres dimensiones 15 categorías; 59 propiedades; 193 indicadores y 611 aspectos por evaluar, tal como se muestra en el siguiente cuadro. 
Cuadro 2

Resumen de los elementos del modelo TAG

\begin{tabular}{|c|c|c|c|c|}
\hline Dimensión & Categorías & Propiedades & Indicadores & $\begin{array}{c}\text { Aspectos a } \\
\text { evaluar }\end{array}$ \\
\hline Tecnología & 5 & 15 & 15 & 54 \\
\hline Aprendizaje & 7 & 15 & 15 & 60 \\
\hline Gestión & 3 & 29 & 163 & 497 \\
\hline Total & $\mathbf{1 5}$ & $\mathbf{5 9}$ & $\mathbf{1 9 3}$ & $\mathbf{6 1 1}$ \\
\hline
\end{tabular}

Fuente: Aguas (2018)

De igual forma, en la fase de planificación se analizó el planteamiento matemático y representación gráfica del modelo TAG especificado a través del trabajo titulado "Tag: Introduction to an Ubiquitous Learning Model to Assess the Ubiquity Level in Higher Education Institutions" (Zea, Aguas, Lalinde, Toro, \& Vieira, 2013) el cual, en términos generales, señala que las tres dimensiones pueden ser vistas como un cubo tridimensional donde se pueden ubicar el nivel de ubicuidad en el cual se encuentran enmarcados los procesos misionales de una IES.

Dado que cada una de las dimensiones se compone de categorías y propiedades, y éstas por indicadores y aspectos a evaluar, según el caso, el valor definitivo del nivel de ubicuidad se determina a través de la aplicación recursiva del modelo matemático sobre cada uno de los ejes del cubo o hipercubo. Es así como, para determinar el nivel de ubicuidad se parte de tres variables ${ }^{4}$ (dimensiones): Tecnología, Aprendizaje y Gestión. El valor de cada una se determina identificando una serie de variables de segundo nivel que la definen y se aplica el modelo para determinar el nivel de la variable original. En este sentido, el modelo puede aplicarse recursivamente tantas veces como sea necesario (Zea Restrepo, Pulido Lalinde, Agudelo, Vieira Mejía, \& Aguas Núñez, 2012) y matemáticamente considera dos casos. El primero, cuando todas las variables tienen el mismo peso, es decir que se pretende que todas las variables tengan el mismo nivel de desarrollo y por ende la contribución de cada variable es igual. Así, siendo un modelo con $n$ variables, para determinar el nivel, se evalúa cada una de las variables $v_{i}, 1 \leq i \leq n$, y se representa en el hiperplano $\mathrm{H}$ trazado por los puntos $p_{n}=\left(0, \ldots, v_{n}, \ldots, 0\right)$ y se identifica $p=\left(v_{1}, v_{2}, \ldots, v_{n}\right) \in H$ tal que $\forall i, j v_{i}=v_{j}$. El nivel se define como $E=\|p\|$.

Así, aplicando el modelo matemático para " $n$ " componentes, se tiene para $P_{1}=\left(V_{1}, 0, \ldots, 0\right) ; P_{2}=\left(0, V_{2}, \ldots, 0\right) ; P_{j}=\left(0, \ldots, 0, V_{j}, 0, \ldots, 0\right) ; 1 \leq j \leq n ; P_{n}=\left(0, \ldots, 0, V_{n}\right.$, con la condición $\mathrm{X}_{1}=\mathrm{X}_{2}=\ldots=\mathrm{X}_{\mathrm{n}}$, se tiene que $E=\|P\|=\frac{\sqrt{n} \prod_{i=1}^{n} V_{i}}{\sum_{j=1}^{n} \prod_{i=1}^{n} V_{i}}$ cuando $V_{j}, \forall_{i}, \forall_{j}$ quedando $E=\frac{\sqrt{n}}{n} V_{i}$ para $P \in H$ con

\section{$\frac{V_{1} m V_{n}}{V_{1-1} V_{n-1}+V_{1} V_{3} \cdots V_{n}+\cdots+V_{2} \cdots V_{n}}$.}

El segundo caso contemplado ocurre cuando las variables tienen peso constante, pero difieren entre sí, en lugar de modificar el modelo matemático se procede a normalizar los ejes. Así, siendo $v_{i}$ y $v_{j}$ las variables de interés con pesos relativos $w_{i}$ y $w_{j}$ se construyen nuevas variables $v_{i}^{r}=v_{i} / w_{i}$ y $v_{j}^{r}=v_{j} / w_{j}$ lo que permite que dichas variables $v_{i}$ y $v_{j}$ tengan el mismo peso relativo y se aplica puede aplicar el modelo tal como se demuestra en el primer caso (Zea Restrepo et al., 2012).

\footnotetext{
${ }^{4}$ Dado que se está abordando la representación matemática de TAG, es importante señalar, que cuando se habla de variables, se está haciendo alusión a las dimensiones (Aprendizaje, Tecnología y Gestión).
} 
La principal restricción del modelo matemático radica en que para que el nivel tenga un valor positivo se requiere que todas las variables sean no nulas, pues con una sola variable que tenga valor cero, el hiperplano pasa por el punto $(0,0, \ldots, 0)$ y por lo tanto el nivel es $E=0$ (Zea Restrepo et al., 2012).

Es así, como en la siguiente figura, y con el propósito de ilustrar en términos generales la estructura del modelo TAG, se presentan las dimensiones, dentro de las cuales se distribuyen las categorías, propiedades, indicadores y aspectos a evaluar que se relacionan en el cuadro 2.

Figura 1

Cubo TAG (Tecnología, Aprendizaje y Gestión)

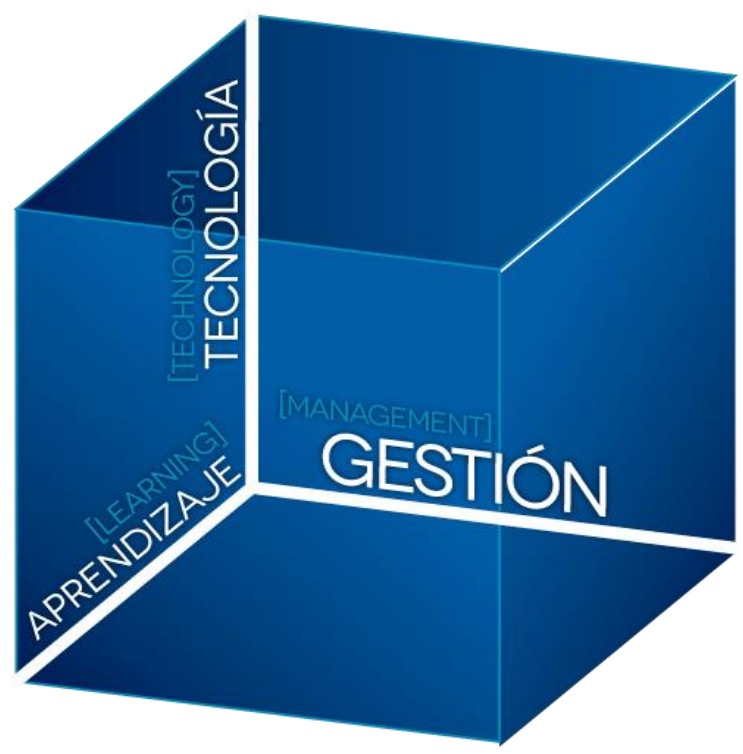

Fuente: Zea et al. (2013)

En virtud de lo anterior y teniendo claros cada uno de los elementos planteados se pudo desarrollar un primer avance del modelo de datos que posee la aplicación, donde la base principal del sistema está dada por las tres dimensiones, las categorías que pertenecen a estas, sus propiedades y los aspectos a valorar respectivos.

De igual forma se planificaron las distintas formas de estimación de los aspectos a valorar, propiedades y categorías de conformidad con los instrumentos y rúbricas desarrollados para el modelo TAG por Aguas (2018). Dichas rúbricas permiten determinar el valor para una propiedad y una vez calculadas todas las propiedades que pertenecen a una categoría se realiza la aplicación de manera recursiva del modelo, logrando el valor de dicha categoría. Así mismo, cuando todas las categorías que pertenezcan a una dimensión posean un valor estimado, la aplicación toma los valores de las categorías y calcula el valor de la dimensión correspondiente.

Con respecto al desarrollo del sistema y debido a que es un aplicativo web, se decidió usar un entorno local donde se utilizaron las siguientes herramientas: xampp como emulador del servidor y Visual Studio Code para la edición de texto y gestión de archivos.

\subsection{Diseño}

En la etapa de diseño se señalan dos actividades principales: diseño de la base de datos y modelamiento de la arquitectura del sistema. En el primer caso, se realizó un modelo relacional con la construcción de una base de datos MySQL. Este cuenta con 16 tablas relacionadas entre sí. Las tablas dimensiones, categorías, propiedades y variables contienen el core de la aplicación, pues en estas tablas está la información necesaria para la implementación del modelo TAG. 
Figura 2

Diseño de base de datos, modelo relacional

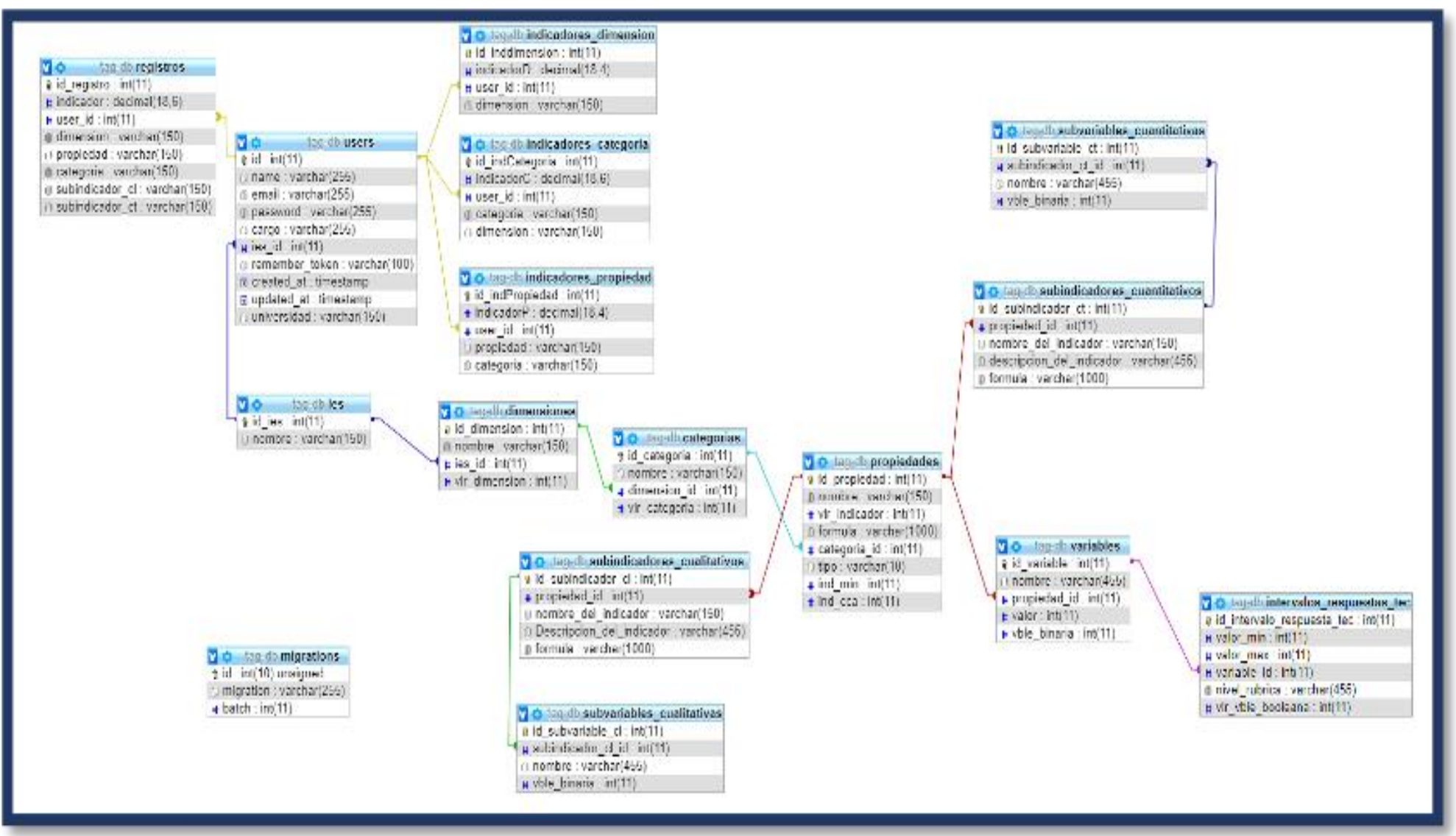

Fuente: elaboración propia

Por otro lado, la aplicación cuenta con un módulo de autenticación de usuarios. Por lo tanto, la información relacionada a los usuarios se encuentra en la tabla "users" y los datos que esta obtiene al utilizar la aplicación se encuentran almacenados en tabla "registros", la cual almacena la evaluación de una rúbrica realizada por el usuario. Las tablas "indicadores_dimension", "indicadores_categoria" e "indicadores_propiedad", son las encargadas de almacenar los indicadores calculados por el modelo. Por su parte, las tablas "subindicadores_cuantitativos" y "subindicadores_cualitativos" se hacen necesarias dependiendo el tipo de propiedad con la que se esté trabajando y almacenan información que permite evaluar indicadores de una propiedad, utilizando además las tablas "subvariables_cuantitativas" y "subvariables_cualitativas".

Para la definición de la arquitectura de la aplicación web se utilizó el framework de desarrollo (back-end) Laravel partiendo de un esquema MVC (Modelo, Vista, Controlador), sin embargo, se incluyeron elementos adicionales como rutas (routes), las cuales son el medio de acceso del usuario a distintas partes de la aplicación y, además, a través de estas se hacen llamadas a los controladores, ya sea para la obtención o envío de datos a la base de datos por medio del controlador. A continuación, en la figura 3, se describe la arquitectura del sistema. 
Figura 3

Arquitectura del sistema

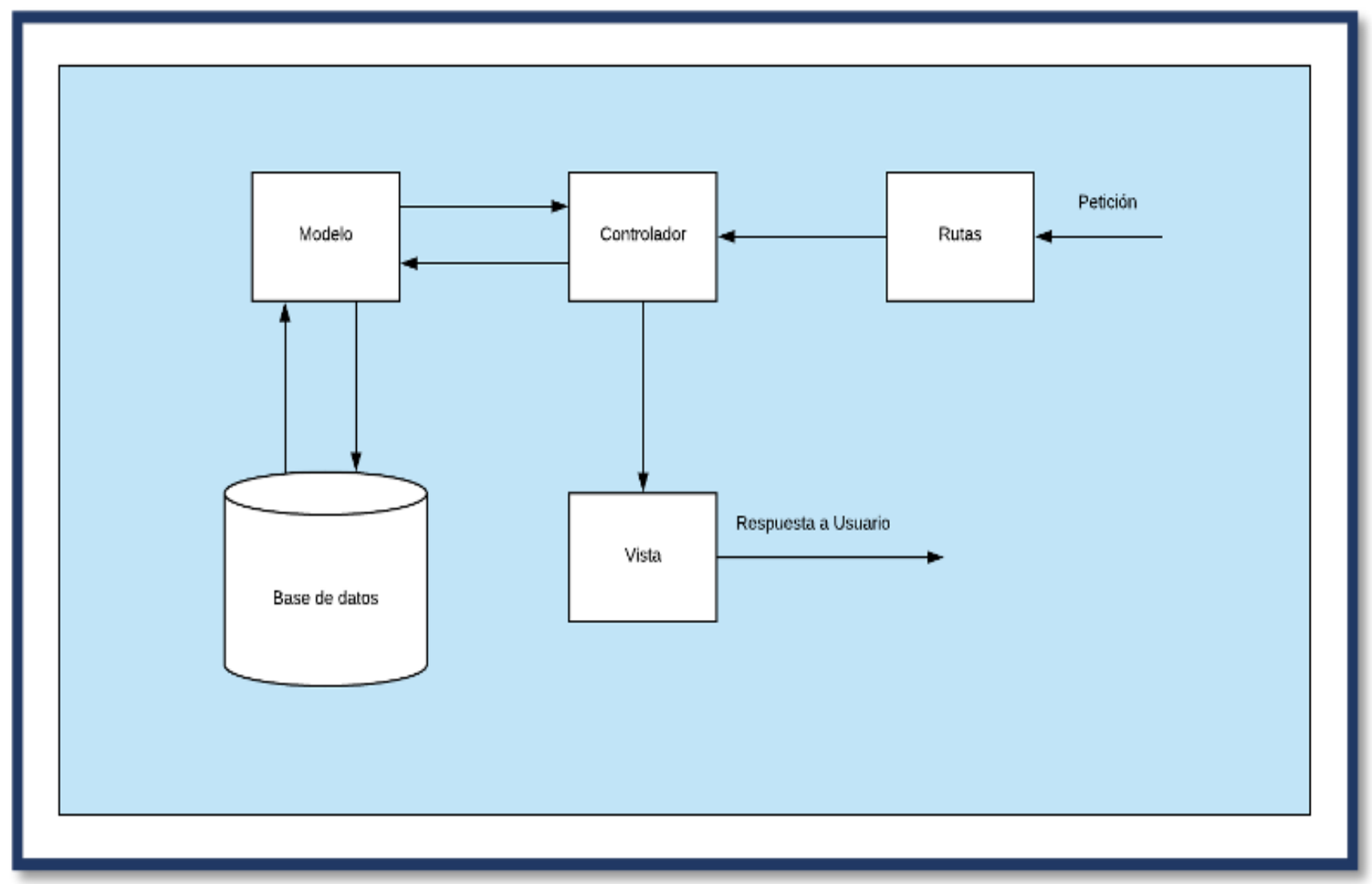

Fuente: elaboración propia

\subsection{Codificación}

En este proceso se definió PHP como lenguaje de programación debido a las facilidades que tiene en el desarrollo, teniendo en cuenta que la aplicación está escrita bajo el framework Laravel. De igual forma, se utilizó Javascript en la realización de funciones referentes a la aplicación y a la interacción con la misma.

Como se ha mencionado anteriormente Laravel es el framework para ejecutar el back-end de la aplicación, Las características que se utilizaron de Laravel en el proyecto fueron: el sistema de rutas, la validación de formularios, el sistema blade, y Artisan como herramienta en la consola de comandos.

Para el caso de front-end se escogió AngularJs para manejar los datos traídos de la base de datos en el lado del cliente, y hacer uso de sus características que permiten la creación de single page application. Por otra parte, también se utilizó Bootstrap como segundo framework front-end para llevar a cabo la parte de estilos de la aplicación.

Cabe anotar que al ser una aplicación que parte de la base del patrón del diseño de MVC, el paradigma de programación utilizado es la programación orientada a objetos, donde la declaración de clases y métodos de estas mismas permiten la definición de los modelos a utilizar en la aplicación, y a su vez la creación de objetos para trabajar con los datos obtenidos. 


\subsection{Pruebas}

En esta última fase se ejecutaron las pruebas de funcionamiento del sistema web para la recolección de datos y aplicación del modelo TAG. De esta forma, se escogen dos Instituciones de Educación Superior (IES) Colombianas de características diferenciales en aspectos como:

- Naturaleza: se selecciona una institución educativa de carácter público (IES1) y una institución educativa de carácter privado regida por preceptos de la religión católica (IES2).

- Modalidad de funcionamiento: considerando la forma en cómo se imparten las clases, una de las instituciones educativas utiliza la modalidad virtual (IES1), mientras que la otra usa la metodología presencial, aunque destacan algunas experiencias con educación a distancia combinando clases virtuales con presenciales (B-Learning) (IES2). Esto conforme a lo que establecen estas instituciones en su Proyecto Educativo Institucional y considerando la información que reposa en el Sistema Nacional de Información de la Educación Superior (SNIES) de Colombia.

- Cobertura: 18.000 estudiantes (IES1) vs 3.000 estudiantes (IES2).

- Aseguramiento de la calidad: la institución pública cuenta con la acreditación institucional de calidad por el Ministerio de Educación Nacional de Colombia, mientras que la privada no tiene este tipo de certificación.

Para la obtención de los datos se utilizaron, varias fuentes de información:

1. Revisión de los informes de gestión, boletines estadísticos y en general los documentos oficiales publicados por las IES.

2. Entrevistas directas realizadas a los encargados de los distintos procesos de la organización relacionados con tecnología, aprendizaje y gestión.

3. Solicitud de información complementaria y de soporte que permita la solidez del modelo al momento de su aplicación.

Con la información necesaria obtenida (variables, indicadores cualitativos y cuantitativos por dimensión e identificación de aspectos relevantes), finaliza esta etapa basada en los instrumentos del modelo TAG.

En este proceso, se implementa la aplicación web para modelar el comportamiento del Modelo TAG, la cual se encuentra disponible en http://cetep.unimagdalena.edu.co/tag. Con esta, se recolectaron y calcularon los 611 aspectos a valorar del modelo para cada IES, permitiendo graficar en un plano tridimensional el nivel de ubicuidad en los procesos misionales de cada una, tal como se presenta en la sección de resultados.

\section{Resultados}

A continuación, se presentan de forma sintética los resultados de cada una de las IES valoradas en el nivel de ubicuidad de sus funciones misionales, de conformidad con las dimensiones del modelo TAG, calculadas a partir de la recolección de las variables, aspectos a valorar, indicadores, propiedades y categorías que las conforman. Cabe anotar que la gestión de dichos datos y su procesamiento se hicieron de conformidad con lo estipulado en la representación matemática del modelo TAG y con la imprescindible ayuda de la aplicación web diseñada para ese fin. 
Cuadro 3

Resumen de valores por dimensión y categoría

\begin{tabular}{|c|c|c|c|c|c|}
\hline \multicolumn{3}{|c|}{ Resultados } & \multirow[t]{2}{*}{ Categoría } & \multirow{2}{*}{$\begin{array}{l}\text { Valor categoría } \\
\text { IES } 1\end{array}$} & \multirow{2}{*}{$\begin{array}{c}\text { Valor categoría } \\
\text { IES } 2\end{array}$} \\
\hline Dimensión & IES 1 & IES 2 & & & \\
\hline \multirow{5}{*}{$\begin{array}{c}\text { T } \\
\text { (Tecnología) }\end{array}$} & \multirow[t]{5}{*}{0.7} & \multirow[t]{5}{*}{0.7} & Seguridad & 4.4 & 4.7 \\
\hline & & & Compatibilidad & 2.6 & 3.7 \\
\hline & & & Computación autónoma & 1.5 & 1.8 \\
\hline & & & Sensado de la situación & 1.5 & 0.9 \\
\hline & & & $\begin{array}{l}\text { Motor de inteligencia } \\
\text { autoformada }\end{array}$ & 0.7 & 1.1 \\
\hline \multirow{7}{*}{$\begin{array}{c}\text { A } \\
\text { (Aprendizaje) }\end{array}$} & \multirow[t]{7}{*}{0.13} & \multirow[t]{7}{*}{0.379} & Procesos de interacción & 0.075135 & 0.24 \\
\hline & & & Transferencia & 4.2426 & 4.24264 \\
\hline & & & $\begin{array}{l}\text { Eliminación de barreras de } \\
\text { tiempo }\end{array}$ & 1.2121 & 5.30330 \\
\hline & & & Espacios de Aprendizaje & 4.2426 & 5.30330 \\
\hline & & & $\begin{array}{l}\text { Aprovechamiento de las } \\
\text { diferencias de los usuarios }\end{array}$ & 0.2734 & 0.9524 \\
\hline & & & Formas de representación & 2.1213 & 5.3033 \\
\hline & & & Sensibilidad al contexto & 1 & 1.00 \\
\hline \multirow{3}{*}{$\begin{array}{c}\text { G } \\
\text { (Gestión) }\end{array}$} & \multirow[t]{3}{*}{0} & \multirow[t]{3}{*}{0} & Procesos estratégicos & 0 & 0.00 \\
\hline & & & Procesos misionales & 0 & 0.00 \\
\hline & & & Procesos de apoyo & 0.5 & 0.50 \\
\hline
\end{tabular}

Fuente: elaboración propia a partir de Aguas (2018)

Al revisar los datos arrojados por la aplicación, es posible notar que los resultados por dimensión evidencian similitudes marcadas entre ambas instituciones en las dimensiones Tecnología y Gestión. En la primera, se muestran resultados similares lo que causa que se obtenga el mismo puntaje para ambas instituciones en esta dimensión, mientras en gestión, es posible afirmar que existen deficiencias marcadas en ambas instituciones, respecto a las variables que plantea el modelo TAG, las cuales fueron valoradas con cero (0) ocasionando que la dimensión fuese valorada de igual forma. Estos resultados pueden ser atribuídos al contexto en el que se desenvuelven las IES, así como a su tamaño, nivel de desarrollo y complejidad.

Además, es importante destacar que, respecto a la divulgación de su información financiera como mecanismo de transparencia en la gestión, la IES1 evidenció ser más propensa a publicar dicha información por diferentes medios, por el contrario, la IES2 maneja dichos temas con suma privacidad y cautela.

Por último, en la dimensión de aprendizaje las IES se diferencian siendo mejor valorada la IES2, esto puede ser debido a que, al tener un enfoque virtual en sus programas académicos, evidencia mejor el desarrollo de modelos pedagógicos centrados en el estudiante potenciando en sus estudiantes la adquisición de competencias en uso de herramientas TIC para fortalecer con ellas competencias genéricas y específicas.

Desde una perspectiva global y considerando el valor máximo posible, se proponen cinco (5) niveles de ubicuidad relacionadas con los procesos misionales de las IES:

1. Nivel nulo, cuando el valor resultante al aplicar el modelo matemático con las tres dimensiones es igual a cero.

2. Nivel bajo, el resultado está entre cero (0) siendo estrictamente mayor que este y $\sqrt{3}$ inclusive.

3. Nivel básico, para valores que sean mayores a $\sqrt{3}$ y menores o iguales a $2 \sqrt{3}$. 
4. Nivel satisfactorio, si el resultado se encuentra entre $2 \sqrt{3}$, siendo estrictamente mayor a este límite y $\left(\frac{\sqrt{3}}{3} * 8,5\right)$ inclusive.

5. Nivel superior, cuando el valor resultante sobrepase $\left(\frac{\sqrt{3}}{3} * 8,5\right)$.

Analizando los resultados obtenidos de la aplicación del modelo con los datos obtenidos de las IES en el estudio, resalta la dimensión de gestión con un resultado de cero (0) en ambas instituciones, como consecuencia de que alguna de las categorías que hacen parte de la dimensión fueron valoradas en cero (0). Esto permite clasificar a ambas IES en el nivel nulo.

Finalmente, con la aplicación web desarrollada, se grafican los niveles de ubicuidad de las funciones misionales de las dos IES seleccionadas, de la siguiente forma.

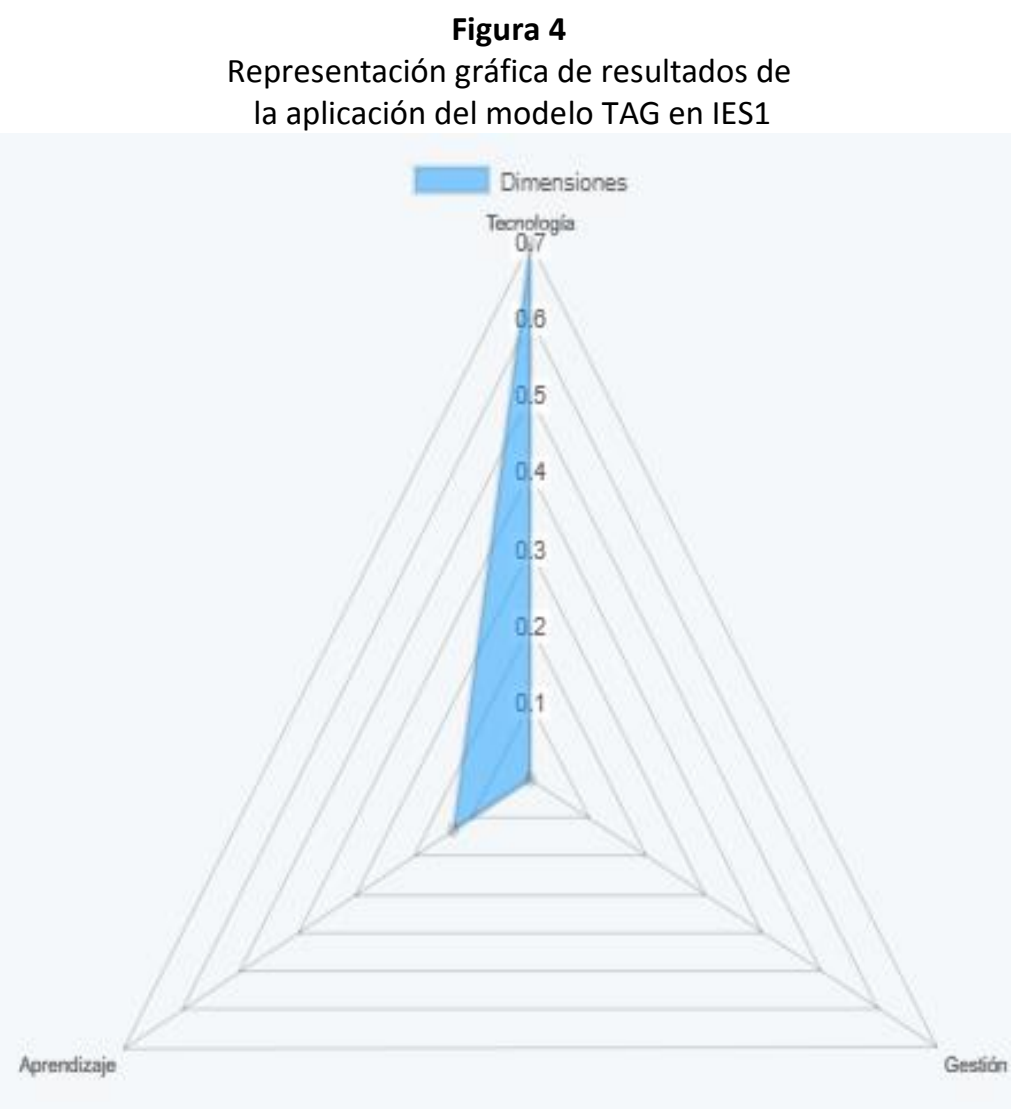

Fuente: elaboración propia. 
Figura 5

Representación gráfica de resultados de

la aplicación del modelo TAG en IES2

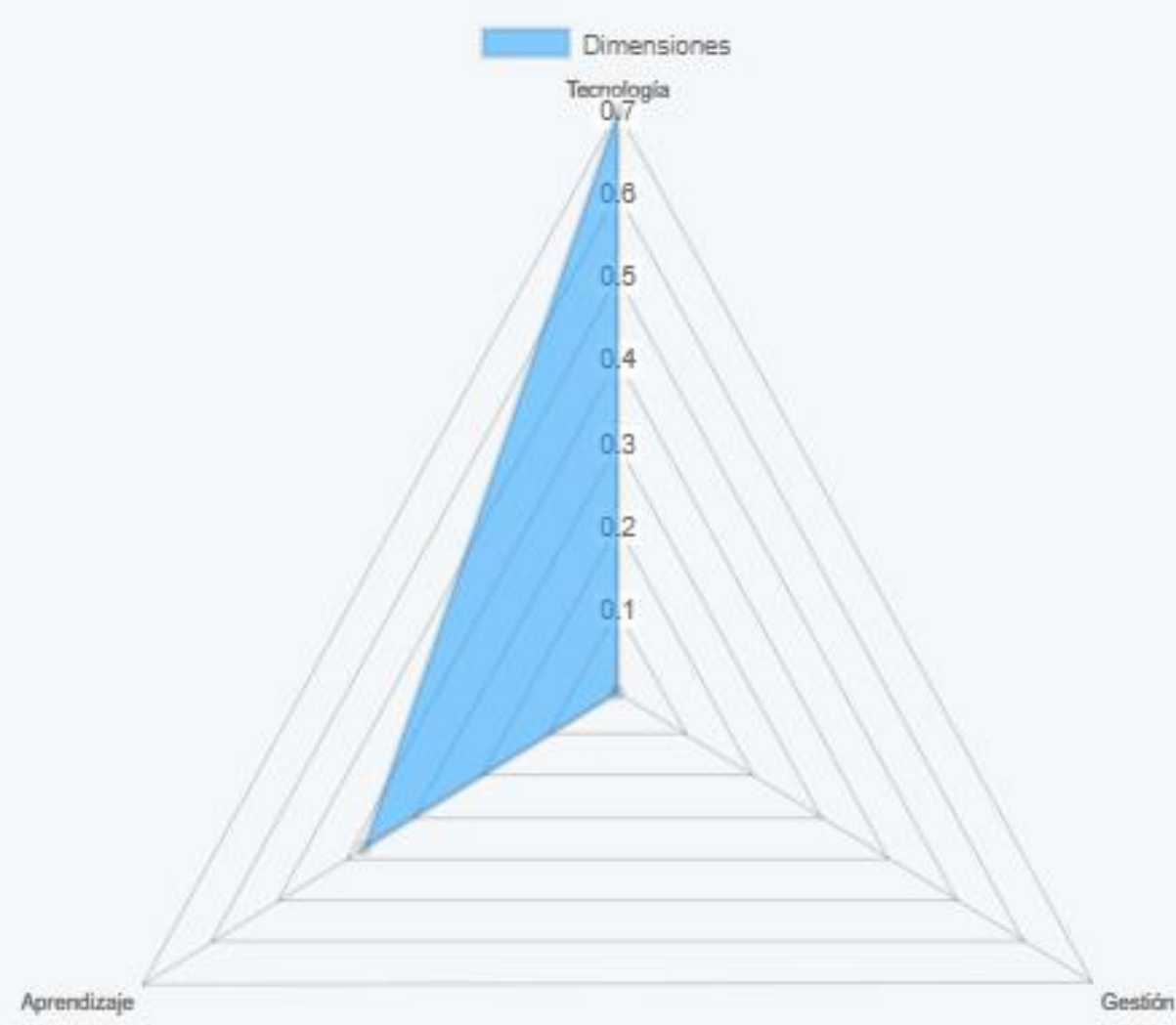

Fuente: elaboración propia

\section{Conclusiones}

Como pudo evidenciarse este artículo sintetiza la necesidad de contar con una herramienta tecnológica capaz de facilitar la recolección de los múltiples datos requeridos por las Instituciones de Educación Superior susceptibles de aplicación del modelo TAG y la versatilidad de que dicha aplicación adaptara los cálculos y procesamiento de dichos datos, de conformidad con las propiedades, categorías y dimensiones establecidas. Es así como, podría concluirse que:

Al hacer uso de la metodología de programación extrema se pudo llevar a cabo el desarrollo de la aplicación en un periodo reducido de tiempo cumpliendo con los requerimientos especificados, esto debido al enfoque de simplicidad que tiene esta metodología, donde la codificación, la realización de pruebas y la integración continua son fundamentales para el desarrollo el proyecto.

Teniendo en cuenta que el equipo de trabajo es de solo tres personas, la utilización de programación extrema supuso una ventaja gracias a las actividades, fases y roles pensados por esta metodología para ser llevados a cabo por un grupo reducido de personas, permitiendo así la comunicación y el conocimiento general del sistema por todo el equipo.

Programación extrema al ser una metodología en la cual se hacen pruebas de forma constante, permitió que el desarrollo del sistema se adaptara a los cambios de requerimientos que se presentaron, haciendo así que las funcionalidades del sistema estuvieran alineadas con los objetivos y requerimientos del proyecto. 
El sistema web desarrollado cumple a cabalidad con los planteamientos y restricciones del modelo TAG, tal como pudo corroborarse en la aplicación del modelo TAG en dos IES colombianas con distintos niveles de complejidad en sus funciones misionales. De igual forma, dicho sistema abre la posibilidad de otras IES más puedan de forma autónoma autoevaluar el nivel de ubicuidad de sus funciones misionales y de esa forma compararse con sus pares en búsqueda de acciones de mejora continua.

La aplicación desarrollada, bajo la metodología XP resulta versátil para posibles mejoras que sean incorporadas al modelo TAG, como por ejemplo la integración de ponderaciones a las variables, aspectos a valorar, propiedades y categorías respectivas.

\section{Referencias bibliográficas}

Aguas, R. (2018). TAG: Modelo Teórico de Valoración del Nivel de Ubicuidad de las Funciones Misionales de una Institución de Educación Superior. Universidad EAFIT. https://doi.org/10.1017/CBO9781107415324.004

Cope, B., \& Kalantzis, M. (2009). Ubiquitous learning: An agenda for educational transformation. Ubiquitous Learning, 1-14.

Duart, J. M., \& Lupiáñez, F. (2004). Procesos institucionales de gestión de la calidad del E-Learning en Instituciones educativas universitarias. Primer Encuentro de Los Miembros de Conaces 8, 1-19. Retrieved from http://www.mineducacion.gov.co/1621/articles-85679_Archivo_pdf.pdf

Fojtik, R. (2011). Extreme programming in development of specific software. Procedia Computer Science, 3, 1464-1468. https://doi.org/10.1016/j.procs.2011.01.032

Salinas, J. (2004). Hacia un modelo de educación flexible: Elementos y reflexiones (pp. 145-170).

Weiser, Marc. (1994). The world is not a desktop. Interactions, 1(1), 7-8. https://doi.org/10.1145/174800.174801

Weiser, Mark. (1993a). Some computer science issues in ubiquitous computing [Algunas cuestiones informáticas en la computación ubicua]. Communications of the ACM, 36(7), 75-84.

Weiser, Mark. (1993b). Ubiquitous computing. Computer, 26(10), 71-72. https://doi.org/10.1109/2.237456

Williams, P. E. (2003). Roles and Competencies for Distance Education Programs in Higher Education Institutions. American Journal of Distance Education, 17(1), 45-57. https://doi.org/10.1207/S15389286AJDE1701_4

Zea, C., Aguas, R., Lalinde, J., Toro, G., \& Vieira, C. (2013). Tag: Introduction to an Ubiquitous Learning Model to Assess the Ubiquity Level in Higher Education Institutions. Journal Ubiquitous Learning, 5(2), 1-15.

Zea, C., \& Atuesta, M. (2007). Hacia Una Comunidad Educativa Interactiva. Fondo Editorial Universidad EAFIT. Retrieved from https://books.google.com.co/books?id=clG-RGXq3q4C

Zea, C., Atuesta, M., \& González, M. (2000). Conexiones: informática y escuela un enfoque global. Editorial Universidad Pontificia Bolivariana. Retrieved from https://books.google.com.co/books?id=OJRMLwAACAAJ

Zea, C., Trujillo, J., Atuesta, M., \& Foronda, N. (2005). Características de los procesos de gestión en los contextos E-Learning. Revista Universidad EAFIT, 41(140), 43-57.

Zea Restrepo, C., Pulido Lalinde, G., Agudelo, O., Vieira Mejía, C., \& Aguas Núñez, R. (2012). Modelo TAG: Referentes para valorar el nivel de ubicuidad en una institución de educación superior.

Esta obra está bajo una Licencia Creative Commons Attribución-NoCommercial 4.0 International 